\title{
$\alpha$-STABLE LIMITS FOR MULTIPLE CHANNEL QUEUES IN HEAVY TRAFFIC
}

Abstract. We consider a sequence of renewal processes constructed from a sequence of random variables belonging to the domain of attraction of a stable law $(1<\alpha<2)$. We show that this sequence is not tight in the Skorokhod $J_{1}$ topology but the convergence of some functionals of it is derived. Using the structure of the sample paths of the renewal process we derive the convergence in the Skorokhod $M_{1}$ topology to an $\alpha$-stable Lévy motion. This example leads to a weaker notion of weak convergence. As an application, we present limit theorems for multiple channel queues in heavy traffic. The convergence of the queue length process to a linear combination of $\alpha$-stable Lévy motions is derived.

1. Introduction. Proving weak convergence in a function space by proving weak convergence of the finite-dimensional distributions and then proving tightness - this is a technique useful in the space of continuous functions and the space of cadlag functions. Nevertheless, there exist sequences of stochastic processes which are of interest in many applications but are not tight. Below we present a sequence of renewal processes which: (i) lies between two sequences of processes that converge weakly in the Skorokhod $J_{1}$ topology to the same limit, (ii) is not tight in the Skorokhod $J_{1}$ topology, (iii) is tight in the Skorokhod $M_{1}$ topology. However, we are able to prove the weak convergence of some useful functionals of this sequence. This leads to a weaker notion of weak convergence. As an application, functional central limit theorems (FCLTs) for multiple channel queues in heavy traffic are derived when the interarrival and service times do not necessarily have the second moment. We consider a queueing system which consists of $p$ independent arrival channels and $r$ independent service channels, where the arrival and service channels are independent. The arriving customers form

2000 Mathematics Subject Classification: Primary 60K25; Secondary 60G52, 60F17. Research supported by KBN under grant 1 H02B 00218 (2000-2001). 
a single queue and they are served in the order of arrival. We investigate two modes of operation for the service channels. We consider the modified queueing system where a waiting customer is assigned to the service channel which can complete his service first and the service channels are not shut off when they are idle. We also consider the standard queueing system where a waiting customer is assigned to the first service channel available and the service channels are shut off when they are idle. Thus the classical $G I / G / s$ system is a special case of our standard system. We shall restrict our attention to systems in heavy traffic and show that the queue length process in the modified and standard queueing systems tends weakly to a linear combination of $\alpha$-stable processes in the Skorokhod $M_{1}$ topology. This approximation is an extension of the work of Iglehart and Whitt [12] where Brownian FCLTs for queues in heavy traffic are derived.

There has been a growing interest in approximations of queueing systems with such alternative conditions in modeling communication networks. Network traffic measurements have exhibited complex stochastic behavior such as heavy-tailed probability distributions, long-range dependence and selfsimilarity. For background and recent related work for single-server queues under such alternative conditions, see Whitt [19] and references therein.

In Section 2 we discuss limit theorems for a renewal process constructed from a sequence of random variables belonging to the domain of attraction of a stable law $(1<\alpha<2)$. Section 3 deals with limit theorems for queues in heavy traffic in the modified queueing systems. In Section 4 we consider the standard queueing system.

2. A weaker notion of weak convergence. Let $D_{\mathbb{R}}=D_{\mathbb{R}}[0, \infty)$, $n \in \mathbb{N}$, be the space of all $\mathbb{R}$-valued right continuous functions on $[0, \infty)$ with limits from the left, endowed with the Skorokhod topology. For the theory of weak convergence in the Skorokhod $J_{1}$ topology see for instance Ethier and Kurtz [9]. The notion of the Skorokhod topologies $\left(J_{1}\right.$ and $\left.M_{1}\right)$ is introduced in Skorokhod [17]; see also Avram and Taqqu [2]. All stochastic processes in this paper are assumed to be in $D_{\mathbb{R}}$.

Let $X_{n}$ and $X$ be stochastic processes with trajectories in $D_{\mathbb{R}}$.

Definition 2.1. A sequence $\left\{X_{n}: n \in \mathbb{N}\right\}$ of stochastic processes is said to converge weakly in the Skorokhod topology to a stochastic process $X$ if for every bounded functional $f$ continuous in the Skorokhod topology,

$$
\lim _{n \rightarrow \infty} \mathbb{E}\left[f\left(X_{n}\right)\right]=\mathbb{E}[f(X)]
$$

In this case one writes $X_{n} \Rightarrow X$.

Let $\mathbf{I}$ be the identity function. The following theorem on random time change (see Whitt [18, Theorem 3.1]) will be useful in our considerations. 
Proposition 2.1. Let $\left\{Z_{n}: n \in \mathbb{N}\right\}$ and $Z$ be processes in $D_{\mathbb{R}}$ and suppose that $Z_{n} \Rightarrow Z$ weakly in $D_{\mathbb{R}}$. Let $\left\{N_{n}: n \in \mathbb{N}\right\}$ be a sequence of processes with non-decreasing and non-negative sample paths such that $N_{n} \Rightarrow \lambda \mathbf{I}, \lambda>0$. For each $n \in \mathbb{N}, Z_{n}$ and $N_{n}$ are assumed to be defined on the same probability space. Then

$$
Z_{n}\left(N_{n}\right) \Rightarrow Z(\lambda \mathbf{I}) \quad \text { weakly in } D_{\mathbb{R}} .
$$

Let $\left\{T_{k}: k \in \mathbb{N}\right\}$ be an iid sequence of non-negative random variables with common distribution function $F$, and for simplicity $E T_{1}=1$, such that

$$
\frac{1}{\varphi(n)} \sum_{k=1}^{[n t]}\left(T_{k}-1\right) \Rightarrow Z_{\alpha, 1}(t)
$$

weakly in the Skorokhod $J_{1}$ topology where $Z_{\alpha, 1}$ is a strictly $\alpha$-stable Lévy motion with skewness parameter $\beta=1$. We assume $1<\alpha<2$. The function $\varphi$ is given by $\varphi(n)=n^{1 / \alpha} L(n)$, where $L$ is slowly varying at infinity. In other words, $F$ is in the domain of attraction of $Z_{\alpha, 1}(1)$.

Let $N$ be a renewal process constructed from $\left\{T_{k}: k \in \mathbb{N}\right\}$, i.e. for $t \geq 0$,

$$
N(t)=\max \left\{k: \sum_{i=1}^{k} T_{i} \leq t\right\},
$$

and set $N_{n}(t)=N(n t) / n$ for $n \in \mathbb{N}$.

Proposition 2.2. Under the above assumptions, $N_{n} \Rightarrow \mathbf{I}$.

Proof. This follows from the strong law of large numbers for renewal processes (see e.g. Resnick [16, p. 190]) and the fact that the trajectories of $N_{n}$ are non-decreasing, which implies the convergence a.s. in the uniform topology.

Let $S_{n}=\sum_{k=1}^{n} T_{k}$ if $n \geq 1$ and $S_{0}=0$, and

$$
\begin{aligned}
& Z_{n}(t)=\frac{S_{[n t]}-[n t]}{\varphi(n)}, \\
& Z_{n}^{\prime}(t)=\frac{S_{[n t]+1}-[n t]}{\varphi(n)}, \\
& Z_{n}^{\prime \prime}(t)=\frac{S_{[n t]}-n t}{\varphi(n)} .
\end{aligned}
$$

Lemma 2.1. $Z_{n} \Rightarrow Z_{\alpha, 1}, Z_{n}^{\prime} \Rightarrow Z_{\alpha, 1}$ and $Z_{n}^{\prime \prime} \Rightarrow Z_{\alpha, 1}$ weakly in the Skorokhod $J_{1}$ topology as $n \rightarrow \infty$.

Proof. The first convergence follows easily from the assumptions. Notice that

$$
Z_{n}^{\prime}(t)=Z_{n}(t+1 / n)+\frac{1}{\varphi(n)} .
$$


Using Proposition 2.1 we find that $Z_{n}(t+1 / n) \Rightarrow Z_{\alpha, 1}(t)$ weakly in the Skorokhod $J_{1}$ topology. Since $1 / \varphi(n)$ tends to 0 we get the assertion for $Z_{n}^{\prime}$. The proof for $Z_{n}^{\prime \prime}$ is similar.

The above lemma and Proposition 2.1 yield

Proposition 2.3. $Z_{n}\left(N_{n}\right) \Rightarrow Z_{\alpha, 1}$ and $Z_{n}^{\prime}\left(N_{n}\right) \Rightarrow Z_{\alpha, 1}$ weakly in the Skorokhod $J_{1}$ topology.

Let $X_{n}$ be the following process:

$$
X_{n}(t)=\frac{n t-N(n t)}{\varphi(n)} .
$$

Lemma 2.2. For every $t \geq 0$ and $n \in \mathbb{N}$,

$$
Z_{n}\left(N_{n}(t)\right) \leq X_{n}(t) \leq Z_{n}^{\prime}\left(N_{n}(t)\right) .
$$

Proof. This follows from (2.3)-(2.5).

Proposition 2.4. Let $f=\left(f_{1}, \ldots, f_{d}\right)$ be an $\mathbb{R}^{d}$-valued functional preserving the double inequality (2.8), that is, either

$$
f_{i}\left(Z_{n}\left(N_{n}\right)\right) \leq f_{i}\left(X_{n}\right) \leq f_{i}\left(Z_{n}^{\prime}\left(N_{n}\right)\right)
$$

or

$$
f_{i}\left(Z_{n}^{\prime}\left(N_{n}\right)\right) \leq f_{i}\left(X_{n}\right) \leq f_{i}\left(Z_{n}\left(N_{n}\right)\right)
$$

a.s. for every $i=1, \ldots, d$, and assume $f\left(Z_{n}\left(N_{n}\right)\right) \Rightarrow f\left(Z_{\alpha, 1}\right)$ and $f\left(Z_{n}^{\prime}\left(N_{n}\right)\right)$ $\Rightarrow f\left(Z_{\alpha, 1}\right)$. Then

$$
f\left(X_{n}\right) \Rightarrow f\left(Z_{\alpha, 1}\right) .
$$

Proof. This follows from the definition of weak convergence in $\mathbb{R}^{d}$.

Propositions 2.4 and 2.3 imply that the finite-dimensional distributions of $X_{n}$ converge weakly to the finite-dimensional distributions of $Z_{\alpha, 1}$. This also implies that, for instance, $\sup _{0 \leq s \leq t} X_{n}(s)$ tends weakly to $\sup _{0 \leq s \leq t} Z_{\alpha, 1}(s)$. Nevertheless, we show that the sequence $\left\{X_{n}: n \in \mathbb{N}\right\}$ is not tight in the Skorokhod $J_{1}$ topology.

TheOREM 2.1. The sequence $\left\{X_{n}: n \in \mathbb{N}\right\}$ given by (2.7) is not tight in the Skorokhod $J_{1}$ topology.

Proof. It is sufficient to prove that

$$
\limsup _{n \rightarrow \infty} P\left\{w_{1}\left(X_{n}, \theta\right)>\eta\right\}>0
$$

for some $\eta$ and every $\theta>0$ where

$$
\begin{aligned}
& w_{N}\left(X_{n}, \theta\right)=\inf \left\{\max w\left(X_{n},\left[t_{i-1}, t_{i}\right)\right):\right. \\
& \left.\quad 0=t_{0}<\ldots<t_{r}=N, \theta \leq t_{i}-t_{i-1} \leq 2 \theta, i \leq r-1, t_{r}-t_{r-1} \leq 2 \theta\right\}
\end{aligned}
$$

and

$$
w\left(X_{n},\left[t_{i-1}, t_{i}\right)\right)=\sup _{t_{i-1} \leq s, t<t_{i}}\left|X_{n}(t)-X_{n}(s)\right|
$$


(see e.g. Jacod and Shiryaev [13]). The law of large numbers shows that

$$
\frac{S_{[n / 2]}}{n} \rightarrow \frac{1}{2} \quad \text { a.s. as } n \rightarrow \infty \text {. }
$$

This implies that

$$
\frac{S_{[n / 2]}}{n}<1 \quad \text { a.s. for } n \geq n_{0}(\omega) .
$$

Then it is clear that

$$
w_{1}\left(X_{n}, \theta\right) \geq \sup _{k \leq n / 2} \frac{1}{2} \frac{T_{k}}{\varphi(n)} \quad \text { if } \sup _{k \leq n / 2} \frac{T_{k}}{n} \leq \theta \text { and } n \geq n_{0}(\omega) .
$$

Note that

$$
\sup _{k \leq n / 2} \frac{T_{k}}{n} \rightarrow 0
$$

in probability as $n \rightarrow \infty$ because

$$
\sup _{k \leq n} \frac{T_{k}}{\varphi(n)} \Rightarrow Y
$$

weakly where $Y$ is a continuous random variable (see e.g. Resnick [15, p. 54]). Thus,

$$
\begin{aligned}
\limsup _{n \rightarrow \infty} P\left\{w_{1}\left(X_{n}, \theta\right)>\eta\right\} & =\limsup _{n \rightarrow \infty} P\left\{w_{1}\left(X_{n}, \theta\right)>\eta, \sup _{k \leq n / 2} \frac{T_{k}}{n} \leq \theta\right\} \\
& \geq P\left\{\liminf _{n \rightarrow \infty}\left\{w_{1}\left(X_{n}, \theta\right)>\eta, \sup _{k \leq n / 2} \frac{T_{k}}{n} \leq \theta\right\}\right\} \\
& \geq P\left\{\liminf _{n \rightarrow \infty}\left\{\sup _{k \leq n / 2} \frac{1}{2} \frac{T_{k}}{\varphi(n)}>\eta, \sup _{k \leq n / 2} \frac{T_{k}}{n} \leq \theta\right\}\right\} \\
& =\lim _{n \rightarrow \infty} P\left\{\sup _{k \leq n / 2} \frac{1}{2} \frac{T_{k}}{\varphi(n)}>\eta\right\}>0
\end{aligned}
$$

by (2.11) and (2.12). This concludes the proof.

However, as mentioned above, the double inequality (2.8) implies the weak convergence of many useful functionals of $X_{n}$. It turns out that one can define a convergence weaker than the weak convergence.

Definition 2.2. A sequence $\left\{X_{n}: n \in \mathbb{N}\right\}$ of real-valued stochastic processes converges to a process $X$ weakly in the sandwich sense if there are two sequences $\left\{Y_{n}: n \in \mathbb{N}\right\}$ and $\left\{Z_{n}: n \in \mathbb{N}\right\}$ of stochastic processes such that

$$
Y_{n}(t) \leq X_{n}(t) \leq Z_{n}(t)
$$

for each $t \geq 0$ a.s. and $Y_{n} \Rightarrow X$ and $Z_{n} \Rightarrow X$ weakly as $n \rightarrow \infty$.

Thus we get the following result. 
Corollary 2.1. Let $\left\{X_{n}: n \in \mathbb{N}\right\}$ be as defined in (2.7). Then $X_{n} \Rightarrow$ $Z_{\alpha, 1}$ weakly in the sandwich sense in the Skorokhod $J_{1}$ topology.

If $x_{n} \in D_{\mathbb{R}}$ is unbounded above, then its generalized inverse function is defined by $x^{-1}(t):=\inf \{u: x(u)>t\}$. We use the following lemma due to Whitt [18].

Lemma 2.3. Let $x_{n} \in D_{\mathbb{R}}(n \in \mathbb{N})$ be unbounded from above, $x_{n}(0) \geq$ 0 , let the sequence $c_{n}$ of real numbers tend to infinity, and let $a>0$. If $c_{n}\left(x_{n}-a I\right) \rightarrow x$ in the Skorokhod $M_{1}$ topology then $c_{n}\left(x_{n}^{-1}-a^{-1} I\right) \rightarrow$ $-a^{-1} x\left(a^{-1} I\right)$ in the Skorokhod $M_{1}$ topology.

The following corollary can be deduced from Theorem $1 \mathrm{~b}$ of Bingham [4].

Corollary 2.2. Let $\left\{X_{n}: n \in \mathbb{N}\right\}$ be as defined in (2.7). Then $X_{n} \Rightarrow$ $Z_{\alpha, 1}$ weakly in the Skorokhod $M_{1}$ topology.

Proof. Using the Skorokhod representation theorem (see e.g. Billingsley [3]) we can assume that (see Lemma 2.1 and (2.6))

$$
Z^{\prime \prime}(t)=\frac{n}{\varphi(n)}\left(\frac{1}{n} \sum_{k=1}^{[n t]} T_{k}-t\right) \rightarrow Z_{\alpha, 1}(t)
$$

with probability 1 in the Skorokhod $J_{1}$ topology. Applying Lemma 2.3 with $c_{n}=n / \varphi(n), x_{n}(t)=n^{-1} \sum_{k=1}^{[n t]} T_{k}$ and using the equality

$$
x_{n}^{-1}(t)=\frac{N(n t)+1}{n}
$$

we get

$$
\frac{N(n t)-n t}{\varphi(n)} \rightarrow-Z_{\alpha, 1}(t)
$$

with probability 1 in the Skorokhod $M_{1}$ topology. Since the weak convergence is a measure property we get the assertion.

REMARK 2.1. In Corollary 2.2 it is not necessary to assume that the process $N$ (see $(2.3)$ ) is constructed from non-negative random variables, but the expectation of $T_{k}$ has to be positive (see Bingham [4]).

Remark 2.2. For $\alpha=2$ the sequence defined in (2.7) tends weakly in the uniform topology to Brownian motion (see Billingsley [3]).

REMARK 2.3. The convergence in the sandwich sense in the Skorokhod $M_{1}$ topology is essentially weaker than the convergence in the Skorokhod $M_{1}$ topology (even the convergence in the sandwich sense in the Skorokhod $J_{1}$ topology does not imply the convergence in the Skorokhod $M_{1}$ topology). Indeed, consider the following functions defined on $[0, \infty)$ :

$$
x(t)= \begin{cases}0 & \text { if } t<1, \\ 1 & \text { if } t \geq 1,\end{cases}
$$




$$
\begin{aligned}
& x_{n}(t)= \begin{cases}0 & \text { if } t<1-1 / n, \\
1 & \text { if } 1-1 / n \leq t<1, \\
0 & \text { if } 1 \leq t<1+1 / n, \\
1 & \text { if } t \geq 1+1 / n,\end{cases} \\
& y_{n}(t)= \begin{cases}0 & \text { if } t<1+1 / n, \\
1 & \text { if } t \geq 1+1 / n,\end{cases} \\
& z_{n}(t)= \begin{cases}0 & \text { if } t<1-1 / n, \\
1 & \text { if } t \geq 1-1 / n\end{cases}
\end{aligned}
$$

Obviously

$$
y_{n}(t) \leq x_{n}(t) \leq z_{n}(t)
$$

for $t \geq 0$, and $y_{n}(t) \rightarrow x(t)$ and $z_{n}(t) \rightarrow x(t)$ as $n \rightarrow \infty$ in the Skorokhod $J_{1}$ topology, but $x_{n}(t)$ does not converge to $x(t)$ in the Skorokhod $M_{1}$ topology.

3. The modified queueing system. Introducing the modified multiple channel queueing systems we follow Borovkov [5] and Iglehart and Whitt [12]. Consider a queueing system which consists of $p$ independent arrival channels and $r$ independent service channels where the arrival and service channels are independent. Customers arrive one at a time in each of $p$ channels and then immediately join a single queue in front of the servers. A waiting customer is assigned to the service channel which can complete his service first and the servers are not shut off when they are idle. Each server has an associated sequence of potential service times. If a server faces continued demand for service, then the actual service times of its successive customers are just these potential service times, and if there is no demand during any potential service time, then the potential service time is ignored and there is no actual service and no departure. After a server has started to work in the absence of demand, the next demand will in general occur in the middle of some potential service time. The remaining portion of that potential service time will be that next customer's actual service time.

The modified queueing system is of interest in its own right and in our considerations it is also a device. The sequence $\left\{U_{n}^{i}: n \in \mathbb{N}\right\}$ of iid random variables represents the interarrival time between the $(n-1)$ th and $n$th customers in channel $i(i=1, \ldots, p)$ and belongs to the normal domain of attraction of a stable law with stability index $1<\alpha_{i} \leq 2$. The sequence $\left\{V_{n}^{j}: n \in \mathbb{N}\right\}$ of iid random variables represents the $n$th potential service time of server $j(j=1, \ldots, r)$ and belongs to the normal domain of attraction of a stable law with stability index $1<\alpha_{p+j} \leq 2$. These sequences are independent, and $E U_{1}^{i}=1 / u_{i}$ and $E V_{1}^{j}=1 / u_{p+j}$ where $i=1, \ldots, p$ and $j=1, \ldots, r$. As a measure of congestion of our system we define the traffic 
intensity $\varrho=\lambda / \mu$ where

$$
\begin{aligned}
& \lambda=\sum_{i=1}^{p} u_{i}, \\
& \mu=\sum_{j=1}^{r} u_{p+j} .
\end{aligned}
$$

We shall restrict our attention to systems in which $\varrho \geq 1$, that is, to the case of queues in heavy traffic.

Thus, we have

$$
\frac{1}{n^{1 / \alpha_{i}}} \sum_{k=1}^{[n t]}\left(U_{k}^{i}-1 / u_{i}\right) \Rightarrow Z_{\alpha_{i}, 1}(t)
$$

for $i=1, \ldots, p$, and

$$
\frac{1}{n^{1 / \alpha_{p+j}}} \sum_{k=1}^{[n t]}\left(V_{k}^{j}-1 / u_{p+j}\right) \Rightarrow Z_{\alpha_{p+j}, 1}(t)
$$

for $j=1, \ldots, r$ where $Z_{\alpha_{k}, 1}, k=1, \ldots, p+r$, are independent stable Lévy processes with skewness parameter $\beta=1$ (note that they are continuous in probability).

We now define renewal processes associated with the arrival channels and servers. Let

$$
A^{i}(t)=\max \left\{n: \sum_{k=1}^{n} U_{k}^{i} \leq t\right\}
$$

for all $t \geq 0$ and $i=1, \ldots, p$, and

$$
S^{j}(t)=\max \left\{n: \sum_{k=1}^{n} V_{k}^{j} \leq t\right\}
$$

for all $t \geq 0$ and $j=1, \ldots, r$. These processes represent the total number of arrivals in the arrival channel $i$ in the time interval $(0, t]$, and the total number of potential service times in the service channel $j$ in the time interval $(0, t]$, respectively. Set

$$
\begin{aligned}
& A(t)=\sum_{i=1}^{p} A^{i}(t), \\
& S(t)=\sum_{j=1}^{r} S^{j}(t)
\end{aligned}
$$

As in Iglehart and Whitt [12], define the modified queue length process $Q^{m}(t)$ as 


$$
Q^{m}(t)=X(t)-\inf _{0 \leq s \leq t} X(s)
$$

where

$$
X(t)=A(t)-S(t)
$$

Then

$$
\begin{gathered}
\frac{A^{i}(n t)-n t u_{i}}{n^{1 / \alpha_{i}}} \Rightarrow u_{i}^{1+1 / \alpha_{i}} Z_{\alpha_{i},-1}(t), \\
\frac{S^{j}(n t)-n t u_{p+j}}{n^{1 / \alpha_{p+j}}} \Rightarrow u_{p+j}^{1+1 / \alpha_{p+j}} Z_{\alpha_{p+j},-1}(t)
\end{gathered}
$$

for $i=1, \ldots, p$ and $j=1, \ldots, r$ in the sense of Definition 2.2, that is, weakly in the sandwich sense in the Skorokhod $J_{1}$ topology and weakly in the Skorokhod $M_{1}$ topology (see Corollary 2.2). Notice that in (3.24) and (3.25) the skewness parameter $\beta$ is -1 . Set

$$
\alpha=\min \left\{\alpha_{1}, \ldots, \alpha_{p}, \alpha_{p+1}, \ldots, \alpha_{p+r}\right\} .
$$

Thus, we can write the double inequality for the process

$$
X_{n}(t)=\frac{X(n t)-(\lambda-\mu) n t}{n^{1 / \alpha}}
$$

to obtain

Proposition 3.1.

$$
X_{n}(t) \Rightarrow Z(t)=\sum_{i=1}^{p} v_{i}^{1+1 / \alpha_{i}} Z_{\alpha_{i},-1}(t)-\sum_{j=1}^{r} v_{p+j}^{1+1 / \alpha_{p+j}} Z_{\alpha_{p+j},-1}(t)
$$

weakly in the sandwich sense in the Skorokhod $J_{1}$ topology and weakly in the Skorokhod $M_{1}$ topology where $v_{k}=u_{k}$ if $\alpha_{k}=\alpha$ and $v_{k}=0$ if $\alpha_{k} \neq \alpha$ and $k=1, \ldots, p+r$ and the processes on the right hand side of (3.28) are independent.

\section{Proposition 3.2.}

$$
X_{n}(t)-\inf _{0 \leq s \leq t} X_{n}(s) \Rightarrow Z(t)-\inf _{0 \leq s \leq t} Z(s)
$$

weakly in the Skorokhod $M_{1}$ topology.

Proof. This follows from Proposition 3.1 and the fact that the functional $f(x)(t)=x(t)-\inf _{0 \leq s \leq t} x(s)$ is continuous in the Skorokhod $M_{1}$ topology (see Whitt [18]).

Set

$$
Q_{n}^{m}(t)=\frac{Q^{m}(n t)-(\lambda-\mu) n t}{n^{1 / \alpha}} .
$$

We now consider the case when $\lambda=\mu$. Then

$$
Q_{n}^{m}(t)=X_{n}(t)-\inf _{0 \leq s \leq t} X_{n}(s)
$$


and Proposition 3.2 implies

Corollary 3.1. If $\varrho=1$ then

$$
Q_{n}^{m}(t) \Rightarrow Z(t)-\inf _{0 \leq s \leq t} Z(s)
$$

weakly in the Skorokhod $M_{1}$ topology.

We now investigate the case $\lambda>\mu$.

Proposition 3.3. If $\varrho>1$ then

$$
Q_{n}^{m}(t) \Rightarrow Z(t)
$$

weakly in the Skorokhod $M_{1}$ topology.

Proof. Using Theorem 4.1 of Billingsley [3] and Proposition 3.1 it suffices to show that $d\left(X_{n}, Q_{n}^{m}\right) \Rightarrow 0$ weakly where $d$ is the uniform metric. Thus, we have to prove that

$$
P\left\{-\inf _{0 \leq s \leq n t} \frac{X(s)}{n^{1 / \alpha}}>\varepsilon\right\} \rightarrow 0
$$

as $n \rightarrow \infty$ and $\varepsilon>0$ because

$$
\sup _{s \leq t}\left|X_{n}(s)-Q_{n}^{m}(s)\right|=-\inf _{0 \leq s \leq n t} \frac{X(s)}{n^{1 / \alpha}} .
$$

Recall that

$$
\frac{X(t)}{t} \rightarrow \lambda-\mu>0
$$

a.s. as $t \rightarrow \infty$ by the strong law of large numbers for renewal processes (see e.g. Resnick [16]). Thus, for any $\delta>0$, there exists a $t_{0}>0$ such that

$$
P\left\{\sup _{t_{0} \leq s}\left|(\lambda-\mu)-\frac{X(s)}{s}\right| \leq \varepsilon\right\} \geq 1-\delta .
$$

Hence

$$
P\left\{\sup _{t_{0} \leq s \leq n t}\left\{-\frac{X(s)}{n^{1 / \alpha}}\right\} \leq \varepsilon\right\} \geq P\left\{\sup _{t_{0} \leq s}\{-X(s)\} \leq 0\right\} \geq 1-\delta
$$

for $n t>t_{0}$. On the other hand,

$$
\sup _{0 \leq s \leq t_{0}}\left\{-\frac{X(s)}{n^{1 / \alpha}}\right\} \leq \sup _{0 \leq s \leq t_{0}} \frac{A(s)+S(s)}{n^{1 / \alpha}} \leq \frac{A\left(t_{0}\right)+S\left(t_{0}\right)}{n^{1 / \alpha}},
$$

which converges to 0 in probability. Since $\sup _{t_{0} \leq s \leq n t}\left\{-X(s) / n^{1 / \alpha}\right\}$ and $\sup _{0 \leq s \leq t_{0}}\left\{-X(s) / n^{1 / \alpha}\right\}$ both converge to 0 in probability, we have (3.33).

4. The standard queueing system. We use the idea of Borovkov (see [5, Section 5]) to define the standard queueing system (see also Iglehart and Whitt [12]). We define the standard system in terms of the same basic sequences of random variables already used for the modified system. Then 
we prove that the distance between the two queue length processes is very small under the assumption that at least one of the interarrival distributions is heavier than all the service distributions.

In order to define the standard system, we should generate the actual service times from the sequence of potential service times. We consider the same sequences of arrivals and potential service times as in Section 3. We construct the actual service time in such a way that this sequence is also iid and is a subsequence of the given potential service times. Thus, if there is still demand for service after a server has just served a customer, then let the next actual service time be just the next random variable in the sequence of potential service times. If there has been no demand before receiving a customer at time $t$, let the next actual service time be the first unused random variable occurring after time $t$ in the sequence of potential service times. This selection procedure provides a subsequence of iid random variables. As before, customers arrive one at a time in each of the $p$ channels and then immediately join a single queue in front of the $r$ servers. They are served in the order of arrival, but by the first idle server, and the service channels are shut off when they are idle.

Let $\theta_{j}(t)$ denote the shift in the service channel $j$ at time $t$, that is,

$$
\theta_{j}(t)=\left(V_{n_{1}}^{j}+\ldots+V_{n_{l}}^{j}\right)-\left(I_{1}^{j}+\ldots+I_{k}^{j}\right)
$$

where $\left\{V_{n_{l}}^{j}: l \in \mathbb{N}\right\}$ is the subsequence consisting of the unused potential service times in the service channel $j$ and $I_{1}^{j}, \ldots, I_{k}^{j}$ are the idle periods up to time $t$ in the service channel $j$, and

$$
V_{n_{1}}^{j}+\ldots+V_{n_{l}}^{j} \geq I_{1}^{j}+\ldots+I_{k}^{j}
$$

but

$$
V_{n_{1}}^{j}+\ldots+V_{n_{l-1}}^{j}<I_{1}^{j}+\ldots+I_{k}^{j} .
$$

For a more detailed description of the shift $\theta_{j}$ see Iglehart and Whitt [12].

Let $Q(t)$ be the process describing the standard queue length at time $t$. Put

$$
Q_{n}(t)=\frac{Q(n t)-(\lambda-\mu) n t}{n^{1 / \alpha}}
$$

where $\alpha$ is defined in (3.26). Recall bounds on the difference between the queue length processes $Q^{m}(t)$ and $Q(t)$ given in Iglehart and Whitt [12]. For every $t \geq 0$ there exists a $t_{0} \leq t$ such that

$$
\begin{gathered}
Q(t) \leq Q^{m}(t)+r+\sum_{j=1}^{r}\left[S^{j}\left(t_{0}+\theta_{j}\left(t_{0}\right)\right)-S^{j}\left(t_{0}\right)\right], \\
Q^{m}(t) \leq Q(t)+\sum_{j=1}^{r}\left[S^{j}\left(t+\theta_{j}(t)\right)-S^{j}(t)\right] .
\end{gathered}
$$


TheOREM 4.1. Let

$$
\alpha<\min \left\{\alpha_{p+1}, \ldots, \alpha_{p+r}\right\}
$$

where $\alpha$ is defined in (3.26). If $\varrho=1$, then

$$
Q_{n}(t) \Rightarrow Z(t)-\inf _{0 \leq s \leq t} Z(s)
$$

If $\varrho>1$, then

$$
Q_{n}(t) \Rightarrow Z(t)
$$

where the process $Z$ is given in (3.28).

Proof. We show that $d\left(Q_{n}, Q_{n}^{m}\right) \Rightarrow 0$ where $d$ is the uniform metric on the interval $[0,1]$. Together with Corollary 3.1 and Proposition 3.3, this implies the desired result.

Using the bounds given in (4.37) and (4.38) we get

$$
\begin{aligned}
d\left(Q_{n}, Q_{n}^{m}\right) & =\frac{1}{n^{1 / \alpha}} \sup _{0 \leq t \leq 1}\left|Q(n t)-Q_{n}^{m}(n t)\right| \\
& \leq \frac{1}{n^{1 / \alpha}} \sup _{0 \leq t \leq 1}\left(r+\sum_{j=1}^{r}\left[S^{j}\left(n t+\theta_{j}(t)\right)-S^{j}(n t)\right]\right) .
\end{aligned}
$$

Thus it suffices to show that

$$
\frac{1}{n^{1 / \alpha}} \sup _{0 \leq t \leq 1}\left[S^{j}\left(n t+\theta_{j}(t)\right)-S^{j}(n t)\right] \Rightarrow 0
$$

for all $j=1, \ldots, r$. Notice that

$$
\sup _{0 \leq t \leq 1} \theta_{j}(n t) \leq \max _{l \leq \widetilde{S}^{j}(n)+1} V_{n_{l}}^{j}
$$

where $\widetilde{S}^{j}$ is the renewal process generated by the subsequence $\left\{V_{n_{l}}^{j}: l \in \mathbb{N}\right\}$ consisting of the unused potential service times in the service channel $j$. Further

$$
\frac{\widetilde{S}^{j}(n)}{n} \rightarrow u_{p+j} \quad \text { a.s. as } n \rightarrow \infty
$$

and

$$
\max _{l \leq n} \frac{V_{n_{l}}^{j}}{n^{1 / \alpha}} \Rightarrow 0 \quad \text { because } \quad \max _{l \leq n} \frac{V_{n_{l}}^{j}}{n^{1 / \alpha_{p+j}}} \Rightarrow Y^{j}
$$

( $Y^{j}$ is a random variable; see e.g. Resnick [15, p. 54]), and $\alpha<\alpha_{p+j}$ from the assumption (4.39). Thus from the above considerations it follows that

$$
\frac{1}{n^{1 / \alpha}} \sup _{0 \leq t \leq 1} \theta_{j}(n t) \Rightarrow 0
$$

and since $1<\alpha<2$ we get

$$
\frac{1}{n} \sup _{0 \leq t \leq 1} \theta_{j}(n t) \Rightarrow 0
$$


for all $j=1, \ldots, r$. From the assumption (4.39), for $j=1, \ldots, r$ we get

$$
\frac{S^{j}(n t)-u_{p+j} n t}{n^{1 / \alpha}} \Rightarrow 0
$$

which means that this last sequence is C-tight, and this implies that for all $\varepsilon>0$ and all $\eta>0$ there exist $0<\delta<1$ and $n_{0}$ such that for all $n \geq n_{0}$,

$$
P\left\{\sup _{t \leq 1, s \leq \delta} \frac{\left|S^{j}(n(t+s))-S^{j}(n t)-u_{p+j} n s\right|}{n^{1 / \alpha}} \geq \frac{\varepsilon}{2}\right\} \leq \eta .
$$

Thus

$$
\begin{aligned}
& P\left\{\sup _{t \leq 1} \frac{\left|S^{j}\left(n t+\theta_{j}(n t)\right)-S^{j}(n t)\right|}{n^{1 / \alpha}}>\varepsilon\right\} \\
& \leq P\left\{\sup _{t \leq 1}\left(\frac{\left|S^{j}\left(n t+\theta_{j}(n t)\right)-S^{j}(n t)-u_{p+j} n \frac{\theta_{j}(n t)}{n}\right|}{n^{1 / \alpha}}+u_{p+j} \frac{\theta_{j}(n t)}{n^{1 / \alpha}}\right)>\varepsilon\right\} \\
& \leq P\left\{\sup _{t \leq 1} \frac{\left|S^{j}\left(n t+\theta_{j}(n t)\right)-S^{j}(n t)-u_{p+j} n \frac{\theta_{j}(n t)}{n}\right|}{n^{1 / \alpha}}>\frac{\varepsilon}{2}\right\} \\
& \quad+P\left\{\frac{u_{p+j}}{n^{1 / \alpha}} \sup _{t \leq 1} \theta_{j}(n t)>\frac{\varepsilon}{2}\right\} .
\end{aligned}
$$

The second term on the right hand side tends to 0 as $n \rightarrow \infty$ by (4.41), and the first term is bounded above by

$$
\begin{aligned}
P\left\{\sup _{t \leq 1} \frac{\left|S^{j}\left(n t+\theta_{j}(n t)\right)-S^{j}(n t)-u_{p+j} n \frac{\theta^{j}(n t)}{n}\right|}{n^{1 / \alpha}}>\frac{\varepsilon}{2} ; \sup _{t \leq 1} \frac{\theta^{j}(n t)}{n} \leq \delta\right\} & \\
& +P\left\{\sup _{t \leq 1} \frac{\theta_{j}(n t)}{n}>\delta\right\} \leq 2 \eta,
\end{aligned}
$$

which follows from (4.42) and (4.44).

Acknowledgments. The author thanks Søren Asmussen for drawing his attention to this subject and for constructive comments.

\section{References}

[1] S. Asmussen, Applied Probability and Queues, Wiley, New York, 1987.

[2] F. Avram and M. S. Taqqu, Weak convergence of sums of moving averages in $\alpha$ stable domain of attraction, Ann. Probab. 20 (1992), 483-503.

[3] P. Billingsley, Convergence of Probability Measures, Wiley, New York, 1968.

[4] N. H. Bingham, Maxima of sums of random variables and suprema of stable processes, Z. Wahrsch. Verw. Gebiete 26 (1973), 273-293.

[5] A. A. Borovkov, Some limit theorems in the theory of mass service, II. Theor. Probab. Appl. 10 (1965), 375-400. 
[6] J. W. Cohen, Heavy-traffic limit theorems for the heavy-tailed GI/G/1 queue, Tech. Rept. Centrum voor Wiskunde en Informatica, 1997.

[7] - The $\nu$-stable Lévy motion in heavy-traffic analysis of queueing models with heavytailed distributions, Tech. Rept. Centrum voor Wiskunde en Informatica, 1998.

[8] J. G. Dai, A fluid-limit model criterion for instability of multiclass queueing networks, Ann. Appl. Probab. 6 (1996), 751-757.

[9] S. N. Ethier and T. G. Kurtz, Markov Processes, Wiley, New York, 1986.

[10] A. Gut, Weak convergence and first passage times, J. Appl. Probab. 12 (1975), 324-332.

[11] -, Stopped Random Walks, Springer, New York, 1988.

[12] D. L. Iglehart and W. Whitt, Multiple channel queues in heavy traffic, I, Adv. Appl. Probab. 2 (1970), 150-177.

[13] J. Jacod and A. N. Shiryaev, Limit Theorems for Stochastic Processes, Springer, New York, 1987.

[14] M. R. Leadbetter, G. Lindgren and H. Rootzén, Extremes and Related Properties of Random Sequences and Processes, Springer, New York, 1983.

[15] S. I. Resnick, Extreme Values, Regular Variation, and Point Processes, Springer, New York 1987.

[16] —, Adventures in Stochastic Processes, Birkhäuser, Boston, 1992.

[17] A. V. Skorokhod, Limit theorems for stochastic processes, Theory Probab. Appl. 1 (1956), 261-290.

[18] W. Whitt, Some useful functions for functional limit theorems, Math. Oper. Res. 5 (1980), 67-81.

[19] - An overview of Brownian and non-Brownian FCLTs for the single-server queue, Queueing Systems 5 (2000), 39-70.

Department of Mathematics

Wrocław University of Economics

Komandorska 118/120

53-345 Wrocław, Poland

E-mail: michna@manager.ae.wroc.pl

Received on 27.6.2002;

revised version on 18.12.2002 